\title{
Identification of novel putative rheumatoid arthritis susceptibility genes via analysis of rare variants Andrew P Morris* ${ }^{*}$, Eleftheria Zeggini ${ }^{2}$ and Cecilia M Lindgren ${ }^{1}$
}

\author{
Addresses: ${ }^{1}$ Genetic and Genomic Epidemiology Unit, Wellcome Trust Centre for Human Genetics, University of Oxford, Roosevelt Drive, \\ Oxford OX3 7BN, UK and ${ }^{2}$ Wellcome Trust Sanger Institute, The Morgan Building, Wellcome Trust Genome Campus, Hinxton, Cambridge \\ CB10 1HH, UK \\ E-mail: Andrew P Morris* - amorris@well.ox.ac.uk; Eleftheria Zeggini - eleftheria@sanger.ac.uk; Cecilia M Lindgren - celi@well.ox.ac.uk \\ *Corresponding author
}

from Genetic Analysis Workshop 16

St Louis, MO, USA 17-20 September 2009

Published: 15 December 2009

BMC Proceedings 2009, 3(Suppl 7):SI3I doi: I0.II86/I753-656I-3-S7-SI3।

This article is available from: http://www.biomedcentral.com/I753-656I/3/S7/SI3 I

(C) 2009 Morris et al; licensee BioMed Central Ltd.

This is an open access article distributed under the terms of the Creative Commons Attribution License (http://creativecommons.org/licenses/by/2.0), which permits unrestricted use, distribution, and reproduction in any medium, provided the original work is properly cited.

\begin{abstract}
Established loci for rheumatoid arthritis (RA), including HLA-DRBI and PTPN22, do not fully account for the genetic component of susceptibility to the disease. One possible source of as yet undiscovered susceptibility genes are those mediated through effects of rare variants. We present a novel method for gene-based genome-wide scans of whole-genome association (WGA) data to identify accumulations of rare variants associated with disease. We apply our method to WGA SNP genotype data obtained from 868 RA cases and II 94 controls. Our results highlight novel putative RA susceptibility genes that have not previously been identified in large-scale WGA studies.
\end{abstract}

\section{Introduction}

The genetic contribution to susceptibility to rheumatoid arthritis (RA) is well established, with a sibling risk ratio of the order of 5 to 10 [1]. The prevalence of the disease in Caucasians is $\sim 0.8 \%$, the risk being three times higher in females than males [2]. The most recognized RA associations are with haplotypes of the HLA-DRB1 locus and variation in PTPN22, both of which have been widely replicated [3-5]. However, these two associations explain only half of the familial aggregation of the disease. More recently, large-scale whole-genomic association (WGA) studies have suggested a number of novel RA susceptibility loci including variation close to both the alpha and beta chains of the IL2 receptor (IL2RA and IL2RB), a single-nucleotide polymorphism (SNP) in linkage disequilibrium with variation at the TRAF1-C5 locus, two independent signals on chromosome 6q23, and a female-specific effect on chromosome 7q32 [6-8]. However, despite these successes, a large proportion of the genetic component of RA susceptibility remains unexplained.

Large-scale WGA studies of thousands of individuals are well powered to detect the modest genetic effects we expect for complex diseases, provided that the functional variants are common (minor allele frequency (MAF) greater than $1-5 \%$, depending on the sample size of the study). Although a number of common variants for complex diseases have now been identified and replicated, it seems unlikely that the common-disease, 
common-variant hypothesis is all encompassing. Instead, some proportion of genetic susceptibility to complex diseases may be attributed to rare variants, potentially acting together within the same functional unit, with a modest joint effect. Such associations cannot be identified through traditional single-SNP analyses because rare variants are poorly captured by WGA genotyping products [9], and are thus underpowered without sample sizes of tens of thousands of individuals which may be practically or financially infeasible.

Here, we used SNP genotype data obtained from 868 RA cases and 1194 unaffected controls of Northern European ancestry using the Illumina HumanHap $550 \mathrm{k}$ BeadChip to identify genes containing accumulations of rare variants associated with disease susceptibility. The log-odds of disease were modeled as a linear function of the proportion of rare SNPs within a gene at which individuals carry at least one copy of the minor allele within a logistic regression framework. Gene-based genome-wide scans for association with RA were performed, fully accounting for the underlying population structure, both with and without adjustment for the effects of the HLA-DRB1 locus, and allowing for sexdifferentiated effects.

\section{Methods}

Consider a sample of unrelated cases and controls typed for SNPs in a gene or small genomic region. Let $n_{i}$ denote the number of rare SNPs (as defined by some predetermined MAF threshold) for which the $i^{\text {th }}$ individual has been successfully genotyped, and let $r_{i}$ denote the number of these SNPs at which they carry at least one copy of the rare variant. We can model the log-odds of disease of the $i^{\text {th }}$ individual in a logistic regression framework, given by

$$
\ln \left(\frac{\pi_{i}}{1-\pi_{i}}\right)=\alpha+\lambda \frac{r_{i}}{n_{i}}+\boldsymbol{\beta} \mathbf{x}_{i}
$$

In this expression, $\mathbf{x}_{i}$ denotes a vector of covariate measurements for the $i^{\text {th }}$ individual, with corresponding regression coefficients $\beta$. The parameter $\lambda$ is the log-odds ratio for an individual carrying a full complement of rare variants compared with an individual carrying none. Thus, we construct a likelihood-ratio test of association of an accumulation of rare variants with disease by comparing the maximized likelihoods of two models via analysis of deviance: i) the null model where $\lambda=0$; and ii) the alternative model where $\lambda$ is unconstrained. The contribution of the $i^{\text {th }}$ individual to the likelihood is weighted by $n_{i}$ to allow for differential call rates between samples.

\section{Results}

Genotypes were reported for 544,892 autosomal and X-chromosome SNPs. A total of 35,760 SNPs were excluded from analysis through application of quality control (QC) filters of low call rate (<97\%) and extreme deviation from Hardy-Weinberg equilibrium (studywide exact $p<5.7 \times 10^{-7}$, except in females only for $\mathrm{X}$-chromosome SNPs). To account for population structure, identity-by-state metrics were calculated for each pair of individuals for every fifth SNP passing QC filters with study-wide MAF greater than $1 \%$, to minimize the effects of linkage disequilibrium, and excluding the MHC to eliminate any bias due to the effect of HLADRB1. Application of multi-dimensional scaling techniques to the resulting matrix of pair-wise identity-by-state statistics generated five axes of genetic variation associated with RA $(p<0.001)$ after adjustment for sex as a covariate. Genes and their boundaries were determined from the UCSC known genes database (build 35), extended by $50 \mathrm{~kb}$ up- and down-stream to incorporate additional functional elements and the promoter region. Each gene was considered independently, irrespective of any overlap in their boundaries. A total of 29,073 rare variants, defined to have a study-wide MAF of less than $5 \%$, were assigned to at least one of 25,501 genes, and thus taken forward for analysis.

Figure 1a presents results of a gene-based genome-wide scan of association of RA with accumulations of rare variants, adjusted for sex and axes of genetic variation as covariates in the logistic regression model, as a function of gene location. Unsurprisingly, the strongest signals of association were observed for accumulations of rare variants in genes in the major histocompatibility complex (MHC), which are likely to be in linkage disequilibrium with HLA-DRB1 risk haplotypes. The strongest signals of association outside of the MHC $\left(p<10^{-4}\right)$ occurred with FRY (furry homolog), PRPS1L1 (phosphoribosyl pyrophosphate synthetase 1-like 1), and ARNTL (ARNT-like protein 1, brain and muscle), each containing accumulations of rare variants that appear to be protective for RA (Table 1).

To account for the effects of the HLA-DRB1 locus, we repeated our analysis by adjusting for the number of shared epitope alleles, in addition to sex and axes of genetic variation, as covariates in the logistic regression model, the results of which are presented in Figure 1b. The strongest signal of association $\left(p=5.4 \times 10^{-6}\right)$ occurred within the class III region of the MHC, in two overlapping genes, PBX2 (pre-B-cell leukemia homeobox 2) and NOTCH4 (Notch homolog 4). These two genes contained the same three rare variants, jointly occurring with an odds ratio of 0.08 (95\% confidence interval 0.030.24 ) for an individual carrying a minor allele at all of 
(a)

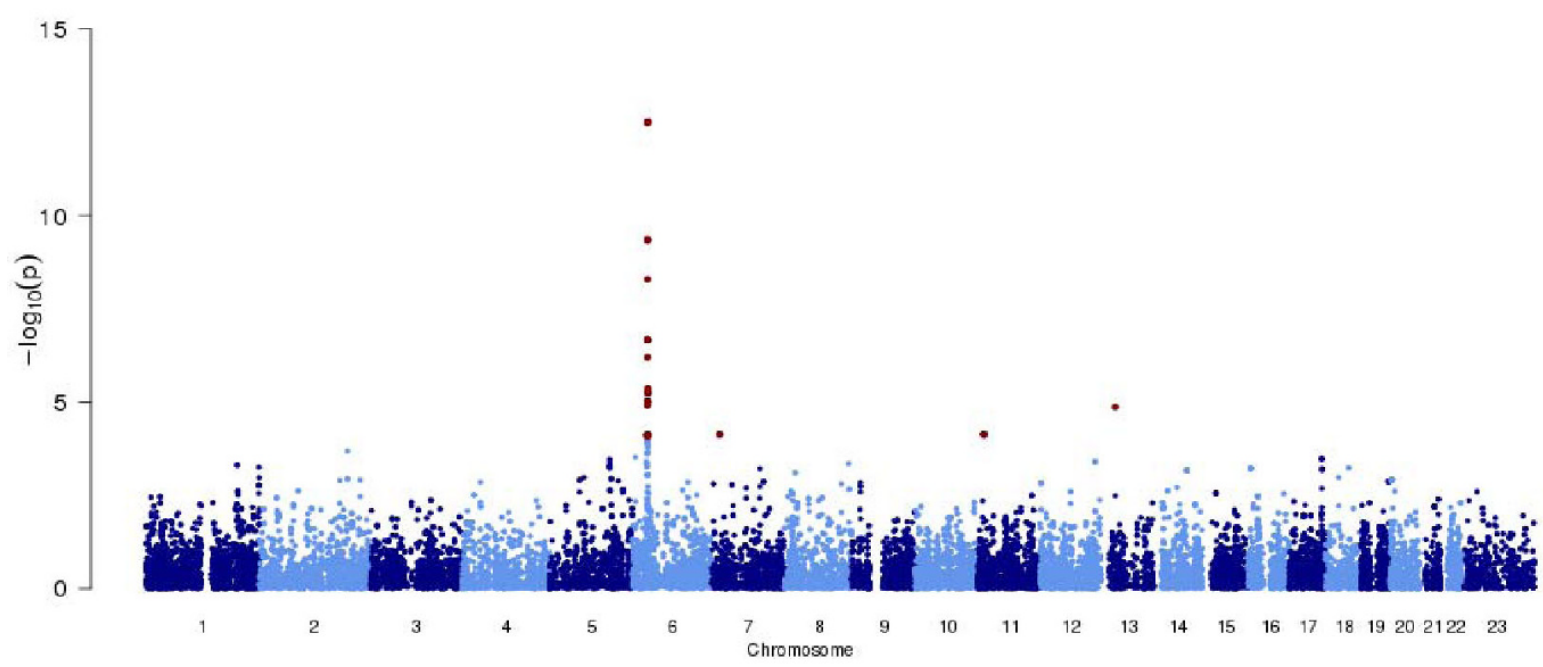

(b)

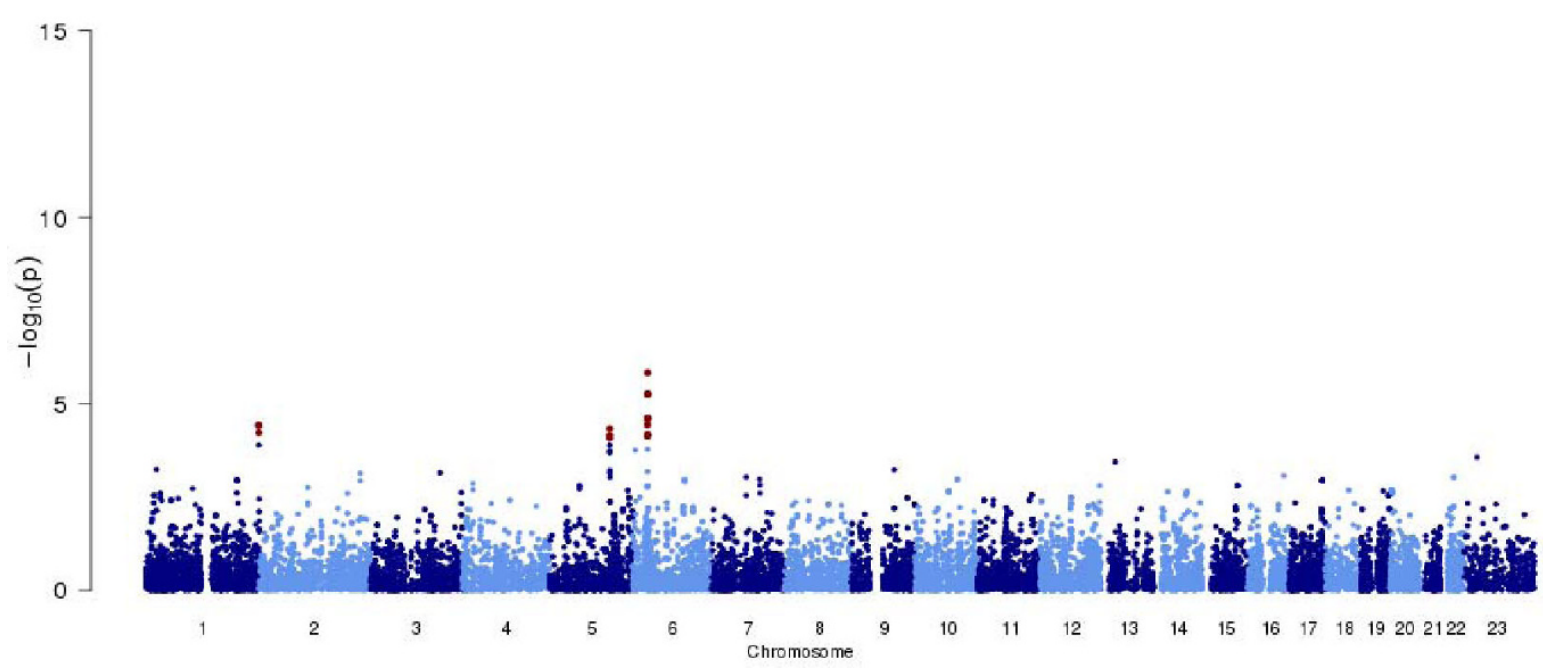

Figure I

Summary of gene-based genome-wide scans of association of RA with accumulations of rare variants. Each genebased test has been adjusted for: (a) sex and five axes of genetic variation; (b) sex, five axes of genetic variation, and the number of shared epitope alleles to account for the effects of HLA-DRBI. Genes achieving a nominal significance threshold of $p<10^{-4}$ are highlighted in red.

Table I: Strongest signals of rare variant association with RA $\left(p<10^{-4}\right)$ outside of the $\mathrm{MHC}^{\mathrm{a}}$

\begin{tabular}{|c|c|c|c|c|c|}
\hline \multirow[b]{2}{*}{ Gene } & \multirow[b]{2}{*}{ Chromosome } & \multicolumn{2}{|c|}{ Rare variants } & \multirow[b]{2}{*}{ Odds ratio $(95 \% \mathrm{Cl})$} & \multirow[b]{2}{*}{$p$-Value } \\
\hline & & Number & Mean MAF (\%) & & \\
\hline FRY & 13 & 5 & 2.6 & $0.10(0.03-0.28)$ & $1.4 \times 10^{-5}$ \\
\hline PRPSILI & 7 & I & 4.4 & $0.46(0.31-0.68)$ & $7.3 \times 10^{-5}$ \\
\hline ARNTLI & 11 & I & 3.0 & $0.38(0.24-0.61)$ & $7.3 \times 10^{-5}$ \\
\hline
\end{tabular}

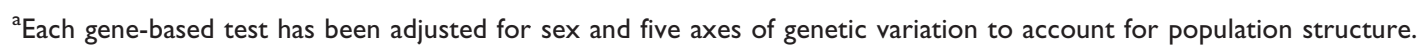


Table 2: Strongest signals of rare variant association with RA $\left(p<10^{-4}\right)$ outside of the MHC, adjusting for the effects of HLA-DRBI

\begin{tabular}{|c|c|c|c|c|c|}
\hline \multirow[b]{2}{*}{ Gene } & \multirow[b]{2}{*}{ Chromosome } & \multicolumn{2}{|c|}{ Rare variants } & \multirow[b]{2}{*}{ Odds ratio $(95 \% \mathrm{Cl})$} & \multirow[b]{2}{*}{$p$-value } \\
\hline & & Number & Mean MAF (\%) & & \\
\hline TRIM58 & 1 & 3 & 1.1 & $0.04(0.01-0.18)$ & $3.7 \times 10^{-5}$ \\
\hline HINTI & 5 & 4 & 3.0 & $0.25(0.13-0.48)$ & $4.6 \times 10^{-5}$ \\
\hline
\end{tabular}

${ }^{a}$ Each gene-based test has been adjusted for sex, five axes of genetic variation to account for population structure, and the number of shared epitope alleles to allow for the effects of HLA-DRBI.

these variants, relative to one carrying none, suggesting them to be protective for RA. This result would indicate RA association with rare variants within the MHC, independent of the effect of HLA-DRB1. Outside of the MHC, the strongest signals of association with RA $(p<$ $10^{-4}$ ) occurred with TRIM58 (tripartite motif-containing 58) and HINT1 (histidine triad nucleotide binding protein 1), again containing accumulations of rare variants that appear to be protective for RA (Table 2).

To test for sex-specific effects, we have performed genebased genome-wide scans of association with RA within males and females separately, with and without adjustments for the effects of the HLA-DRB1 locus. Sexdifferentiated tests of association were performed by summing the deviances obtained across the two sexes, adjusted for axes of genetic variation. The strongest signal of association, not identified in the primary analyses described above, occurred in MYO1B (myosin $1 \mathrm{~B})$, containing six rare variants $\left(p=7.8 \times 10^{-5}\right.$ without adjustment for the effects of HLA-DRB1). There is some evidence of heterogeneity of the effects of accumulations of rare variants in the gene between the sexes $(p=0.074)$. There is a signal of association with RA in females $(p=$ $\left.1.0 \times 10^{-4}\right)$, but no such effect is seen in males $(p=0.45)$, although this may reflect a lack of power due to lower sample size.

\section{Discussion}

We have presented the results of novel methodology for gene-based genome-wide scans for association of RA with an accumulation of rare variants. The strongest signals of association were observed for genes in the $\mathrm{MHC}$, where an accumulation of rare variants reduces risk of RA, presumably as a result of linkage disequilibrium with HLA-DRB1 haplotypes. There are no rare variants $(\mathrm{MAF}<5 \%)$ on the Illumina HumanHap $550 \mathrm{k}$ beadchip passing our QC filters in PTPN22. Rare variants are represented in the other established RA loci (TRAF1$C 5, I L 2 R A$, and IL2RB), but our results do not indicate any evidence of rare variant effects within these genes.

Our results highlight rare variant associations with RA within the MHC, independent of the effects of the HLADRB1 locus. Furthermore, a number of novel putative RA susceptibility genes have been identified outside of the MHC $\left(p<10^{-4}\right)$, with signals of association at least as strong as would be observed through application of traditional single-SNP methods. It is interesting to note that for all of these genes, accumulations of rare variants are associated with decreased risk of RA, suggesting them to be protective. This could reflect the fact that we are able to identify more rare variants in the larger sample of controls than cases, and hence that our analysis has greater power to detect protective associations. We repeated our analyses using rare variant thresholds of MAF less than $2 \%$ and less than $1 \%$. These analyses highlighted no novel susceptibility genes for RA $\left(p<10^{-4}\right)$, reflecting the scarcity of variants with MAF less than $2 \%$ on the Illumina HumanHap 550 k Beadchip (just 15,093 SNPs covering 18,447 genes).

Our analyses highlighted associations with a just a single rare variant in each of the genes PRPS1L1 and ARNTL1. Application of traditional single-SNP analysis would thus produce a similar signal of association to that identified here. However, the advantage of a gene-based analysis is that there is a lesser burden of multiple testing, so that a less stringent genome-wide significance level would be sufficient. The definition of such a threshold is difficult here because each gene is treated as independent, despite the fact that many overlap. The results of overlapping genes will be strongly correlated because they share SNPs in common, and thus a simple Bonferroni correction for the number of tests performed will be highly conservative. Genotypes are more difficult to call at rare variants than more common SNPs, the distinction between heterozygotes and rare homozygotes being less transparent. The QC filters implemented here will exclude the worst offenders, but careful visual inspection of the cluster plots of the remaining variants in associated genes is essential. These two issues highlight the importance of confirmation of these findings in independent replication cohorts, or through meta-analysis with other RA WGA studies, such as that carried out as part of the Wellcome Trust Case Control Consortium [7]. Ideally, studies will be performed in the same population because we expect the spectrum of rare variants to be more variable that common SNPs between even closely related populations. In addition, it would be preferable 
for samples to be typed using the same technology because different panels of rare variants appear on different genotyping products, and are more difficult to impute across platforms than common SNPs [9].

Analysis of rare variants on WGA genotyping arrays is far from ideal, given their scarcity and poor coverage by common SNPs. However, with the increasing availability of large-scale re-sequencing data, such as that generated by the 1,000 Genomes Project [10], we are entering an exciting period for rare variant discovery in which development of analytical strategies to maximize the potential of these investments will be of critical importance.

\section{Conclusion}

We have presented the results of an application of a new method for gene-based genome-wide association of RA with accumulations of rare variants $(\mathrm{MAF}<5 \%)$. Our results confirm strong signals of association for genes in the MHC, and highlight putative novel RA associations which require follow-up in independent replication cohorts from the same population.

\section{List of abbreviations used}

MAF: Minor allele frequency; MHC: Major histocompatability complex; QC: Quality control; RA: Rheumatoid arthritis; SNP: Single-nucleotide polymorphism; WGA: Whole-genome association.

\section{Competing interests}

The authors declare that they have no competing interests.

\section{Authors' contributions}

APM performed the statistical analysis and drafted the manuscript. All authors conceived of the study and read and approved the final manuscript.

\section{Acknowledgements}

The Genetic Analysis Workshops are supported by $\mathrm{NIH}$ grant ROI GM03 I575 from the National Institute of General Medical Sciences. APM and EZ acknowledge funding from the Wellcome Trust. CML is a Scientific Leadership fellow with Nuffield Department of Medicine, University of Oxford.

This article has been published as part of BMC Proceedings Volume 3 Supplement 7, 2009: Genetic Analysis Workshop 16. The full contents of the supplement are available online at http://www.biomedcentral.com/ $|753-656| / 3$ ? issue $=$ S7.

\section{References}

I. Wordsworth $\mathrm{P}$ and Bell J: Polygenic susceptibility in rheumatoid arthritis. Ann Rheum Dis I991, 50:343-346.

2. Brennan $P$ and Silman $A$ : Why the gender difference in susceptibility to rheumatoid arthritis. Ann Rheum Dis 1995 , 54:694-695.
3. Gregersen PK, Silver J and Winchester RJ: The shared epitope hypothesis. An approach to understanding the molecular genetics of susceptibility to rheumatoid arthritis. Arthritis Rheum 1987, 30:1205-1213.

4. Begovich $A B$, Carlton VE, Honigberg LA, Schrodi SI, Chokkalingam AP, Alexander HC, Ardlie KG, Huang Q, Smith AM, Spoerke JM, Conn MT, Chang M, Chang SY, Saiki RK, Catanese JJ, Leong DU, Garcia VE, McAllister LB, Jeffery DA, Lee AT, Batliwalla F, Remmers E, Criswell LA, Seldin MF, Kastner DL, Amos Cl, Sninsky J and Gregersen PK: A missense single-nucleotide polymorphism in a gene encoding a protein tyrosine phosphatase (PTPN22) is associated with rheumatoid arthritis. Am J Hum Genet 2004, 75:330-337.

5. Hinks A, Eyre S, Barton A, Thomson W and Worthington J: Investigation of genetic variation across PTPN22 in UK rheumatoid arthritis (RA) patients. Ann Rheum Dis 2006, 66:683-686.

6. Plenge RM, Seielstad M, Padyukov L, Lee AT, Remmers EF, Ding B, Liew A, Khalili H, Chandrasekaran A, Davies LR, Li W, Tan AK, Bonnard C, Ong RT, Thalamuthu A, Pettersson S, Liu C, Tian C, Chen WV, Carulli JP, Beckman EM, Altshuler D, Alfredsson L, Criswell LA, Amos Cl, Seldin MF, Kastner DL, Klareskog L and Gregersen PK: TRAFI-C5 as a risk locus for rheumatoid arthritis - a genomewide study. N Engl J Med 2007, 357: I199-1209.

7. The Wellcome Trust Case Control Consortium: Genome-wide association study of 14,000 cases of seven common diseases and 3,000 shared controls. Nature 2007, 447:66I-678.

8. Plenge RM, Cotsapas C, Davies L, Price AL, de Bakker PI, Maller J, Pe'er I, Burtt NP, Blumenstiel B, De Felice M, Parkin M, Barry R, Winslow W, Healy C, Graham RR, Neale BM, Izmailova E, Roubenoff R, Parker AN, Glass R, Karlson EW, Maher N, Hafler DA, Lee DM, Seldin MF, Remmers EF, Lee AT, Padyukov L, Alfredsson L, Coblyn J, Weinblatt ME, Gabriel SB, Purcell S, Klareskog L, Gregersen PK, Shadick NA, Daly MJ and Altshuler D: Two independent alleles at 6 q23 associated with risk of rheumatoid arthritis. Nat Genet 2007, 39:|477-|482.

9. Anderson CA, Pettersson FH, Barrett JC, Zhuang JJ, Ragoussis J, Cardon LR and Morris AP: Evaluating the effects of imputation on the power, coverage, and cost-efficiency of genome-wide SNP platforms. Am J Hum Genet 2008, 83: I I2-II9.

10. 1000 Genomes: A Deep Catalog of Human Genetic Variation. http://www. I000genomes.org.

\section{Publish with Bio Med Central and every} scientist can read your work free of charge

"BioMed Central will be the most significant development for disseminating the results of biomedical research in our lifetime. "

Sir Paul Nurse, Cancer Research UK

Your research papers will be:

- available free of charge to the entire biomedical community

- peer reviewed and published immediately upon acceptance

- cited in PubMed and archived on PubMed Central

- yours - you keep the copyright
BioMedcentral 